\title{
Based on PSO cloud computing service discovering
}

\author{
Shen jia jie, chen zhi yun \\ Computer center of School of Information Science \& Technology \\ East china normal university \\ Shanghai, china, 18817519704 \\ E-mail: 51111211010@ecnu.cn
}

\begin{abstract}
Aiming to problem how to discover service which user wants in cloud computing situation, using method of modeling and theoretical analysis, an improved PSO (particle swarm optimization) algorithm is designed to handle this problem. Though theoretical derivation, the correctness of improved PSO algorithm is proofed. The correctness of theoretical derivation and the improved PSO algorithm is also verified by the experiment.
\end{abstract}

Key-word: service discover, PSO algorithm, cloud computing

I Introduction

With the development of cloud computing, more and more application and work is moved from local computer to the cloud computing side. How to searching the cloud computing situation remains a problem to be discussed.

In the paper an improved PSO algorithm is designed to handle the services discover problem under cloud computing situation. Though theoretical derivation, The correctness of the improved PSO algorithm is proofed. The correctness of theoretical derivation and the algorithm is verified by the experiment.

The paper is organized as follow, In second section, the improved Standard PSO algorithm and cloud computing services discover problem will be introduced. In third section, improved PSO algorithm will be presented to handle center base location problem. In fourth section, the conclusion of the paper will be presented.

II Standard PSO algorithm and cloud computing services discover problem

\subsection{Standard PSO algorithm}

Standard PSO algorithm is first presented by Kennedy, Eberhart in $1995^{[1]}$. After standard PSO algorithm, there is a lot of improvement, and several application and case have already been solved by the PSO algorithm.

\subsection{1 the function of standard PSO algorithm}

The position and velocity of swarm as follow:

$$
\left\{\begin{array}{l}
X_{\mathrm{i}}=\left(x_{i 1}, x_{i 2}, . ., x_{i \mathrm{D}}\right) \\
V_{i}=\left(\mathrm{v}_{\mathrm{i} 1}, \mathrm{v}_{\mathrm{i} 2}, . ., \mathrm{v}_{\mathrm{iD}}\right)
\end{array}(1 \leq i \leq n)\right.
$$

The optimization value of the particle $i$ is marked as:

$$
p_{i}=\left(x_{i 1}, x_{\mathrm{i} 2}, \ldots, x_{i n}\right)
$$

The optimization value of the swarm is marked as:

$$
p_{\mathrm{gi}}=\left(x_{\mathrm{gi} 1}, x_{\mathrm{gi} 2}, . ., x_{\mathrm{gin}}\right)
$$

Each particle will change position and velocity the every step based on the follow function:

$$
\begin{aligned}
\mathrm{v}_{i d}^{k+1} & =\omega v_{i d}^{k}+c_{1} \xi\left(p_{i d}^{k}-x_{i d}^{k}\right)+c_{2} \eta\left(p_{g d}^{k}-x_{i d}^{k}\right) \\
x_{i d}^{k+1} & =x_{i d}^{k}+v_{i d}^{k+1}
\end{aligned}
$$

\subsubsection{The description of the standard PSO algorithm}

The step of standard PSO algorithm as follow:

Step 1: initialize the swarm include the position and velocity

Step 2: using the PSO algorithm to find the optimization value.

Step 2.1: find the optimization value of each particle $\left(p_{i}\right)$

and optimization value of $\operatorname{swarm}\left(p_{i g}\right)$.

Step 2.2: update the position and velocity based on function 4.

Step 2.3: calculate the target function value of each particle.

Step 2.4: if step number bigger than maximum step number or the optimization value is reached go to step 3 , else go to step 2.1.

Step 3: Print the optimization value

The improved PSO algorithm have been presented 
by vary papers ${ }^{[2]-[13]}$, for example how to combine the PSO algorithm with SVM algorithm with optimization parameters and how to use different kind of improved PSO algorithm to solve vary problems, in other case the PSO algorithm is designed to handle discrete problem, the binary PSO algorithm is presented. the main different between the standard PSO algorithm and binary PSO algorithm as follows:

The each particles is only include $\{0,1\}$, and the each particles is calculate as follows:

$$
v_{i d}^{k+1}=\omega v_{i d}^{k}+c_{1} \xi_{1}\left(p_{i d}^{k}-x_{i d}^{k}\right)+c_{2} \xi_{2}\left(p_{g d}^{k}-x_{i d}^{k}\right)
$$

$x_{i d}^{k}$ is the step $k$ particle $i$

$$
x_{i d}^{k}=\left\{\begin{array}{l}
1, \operatorname{rand}(0,1)<\operatorname{Sig}\left(v_{i d}^{k}\right) \\
0, \text { else }
\end{array}\right.
$$

$v_{i d}^{k}$ is velocity which is calculated by equation 5 .

\section{2 the problem of the cloud computing services discover}

The network service discover is problem of how to find the services in the computer network. The cloud computing is new organization way of distribution system, how to quick find the services in cloud computing ${ }^{[15]}$ situation is remain a question to discussed.

\section{Improved PSO algorithm}

\section{1 the assumption and definition of the algorithm}

The assumption and definition of the algorithm as follows:

Assumption 1: the cloud computing node's service probability is updated according to time, but the probability of the cloud computing services can be evaluated.

Assumption 2: the algorithm has some target service, and the particles give is information to other particles.

Assumption 3: the PSO algorithm's step number is enough to find target service.

Definition 1: the algorithm searching target services is called as target services, marked as:

$$
t_{i}=\text { service }_{i} \text {, if } \text { service }_{i} \text { is target service }(
$$

Definition 2: To each kind of service every services point has a probability to have this services in it and services points round it is called services exist probability, marked as

$$
s p_{i j}=p_{i j}
$$

$p_{i j}$ is particles the service $i$ in node $j$.

Definition 3: the different between the number of services nodes and all nodes is called as service occupy rate, marked as:

$$
C P=\frac{n_{s}}{n_{a}}
$$

$n_{s}$ is the number of the services node, $n_{a}$ is the number of node.

Definition 4: the different number between correct particles and all particles is called as correct rate of services' searching, marked as:

$$
C R=\frac{n_{f}}{n_{a}}
$$

$n_{f}$ is particles which is found its target service, $n_{a}$ is total particles' number.

\section{2 the step of improved PSO algorithm}

The step of improved PSO algorithm as follows:

Step 1: initialize the swarm include the position and velocity

Step 2: using the PSO algorithm to find the optimization value.

Step2.1: To each particle find the best probability of $p_{i}$ and $p_{g d}$.

Step2.2: update the position(cloud node id) and velocity based on function 5 and function 6 .

Step2.3: calculate the target function value of each particle

Step2.4: if step number bigger than maximum step number or the optimization value is reached go to step 3 ,else go to step 2.1 . 
Step 3: Print the optimization value and service's node

\subsection{The characters of the improved RPCL algorithm}

Theorem 1: In the same algorithm situation, with increasing of the services kind number, the correct of services' searching will be decreasing.

Proof:

Suppose the initial problem the algorithm should find $n$ different kinds of services, the algorithm will use execute time $t$.

The second case, the algorithm should searching $n+k$ different kinds of services, in order to find $n$ different service like first case the algorithm also will can cost time $t$, and then the algorithm will spend some to find the leave $k$ target services. So in the same time e correct of services' searching will be decreasing.

Theorem 2: In the same algorithm situation, with increasing of the particles number, the correct of services' searching will be increasing.

Proof:

With increasing of the increase of the particles' number, the information of each particles each round will increases and the particles number increase the particles will searching the same area in less time, so with the increasing of the particles number, the correct of services' searching will be increasing.

\section{Experiment}

Experiment of this paper is organized as follows, To each Experiment round the PSO algorithm executes 1000 steps. Each experiment data will be run 10 times in different particle number and target service numbers. Table 1 shows the experiment data of the paper:

Table 1 the experiment data of the paper

\begin{tabular}{|l|l|l|l|}
\hline Cloud id & $\begin{array}{l}\text { Services } \\
\text { kind }\end{array}$ & $\begin{array}{l}\text { Cloud point } \\
\text { number }\end{array}$ & $\begin{array}{l}\text { Services } \\
\text { occupy }\end{array}$ \\
\hline 1 & 5 & 20 & $80.00 \%$ \\
\hline 2 & 10 & 50 & $90.00 \%$ \\
\hline 3 & 20 & 100 & $97.00 \%$ \\
\hline 4 & 30 & 150 & $97.33 \%$ \\
\hline 5 & 40 & 200 & $97.00 \%$ \\
\hline 6 & 50 & 300 & $97.00 \%$ \\
\hline
\end{tabular}

As table 1 show, in order to find the characteristic of algorithm, the different experiment is vary from the small to big. Following contents shows the result of the experiment:

Figure 1 shows the relationship between target services number and correct rate of services' searching in different test sets

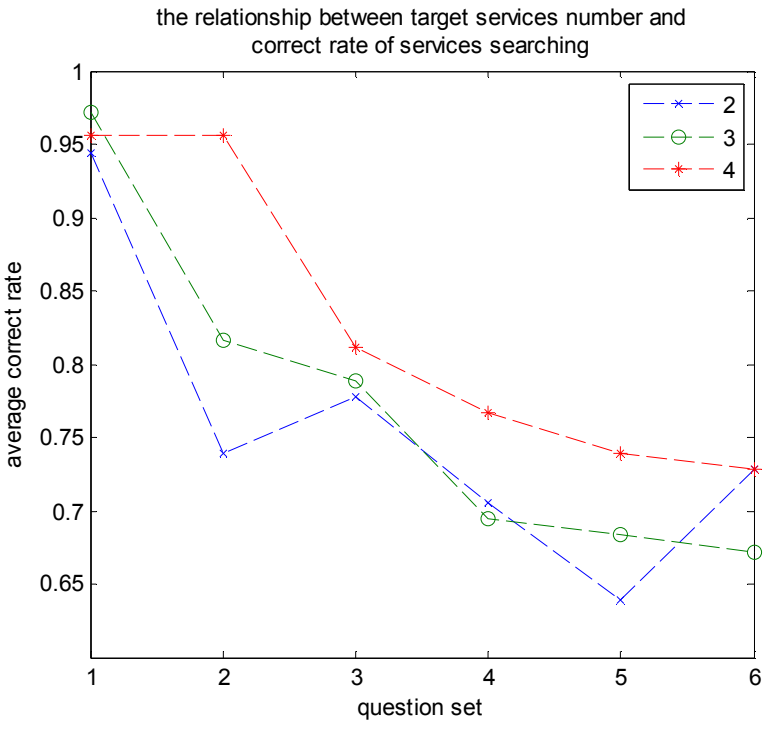

Figure 1 the relationship between target services number and correct rate of services' searching

As figure 1 show, with the increasing of the target services number, the correct rate of services' searching is decreasing. It fits the theorem 1.

Figure 2 shows PSO's particles' number and correct rate of services's searching in different test sets:

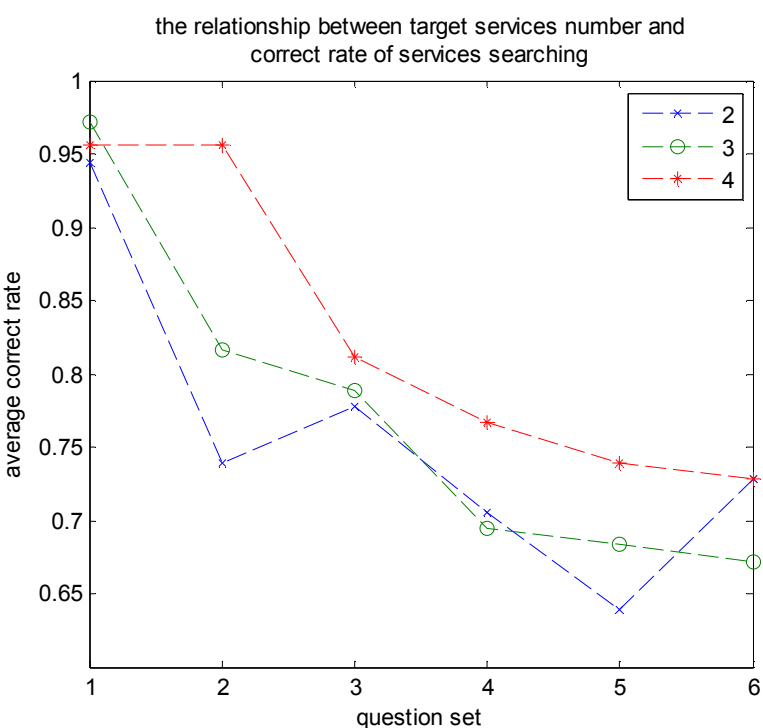

Figure 2 the relationship between PSO's particles' number and correct rate of services' searching

As figure 2 presented, in the same target services number, with the increase of the particle number, the correct rate of services' searching is also increasing. It 
fits the theorem 2.

Figure 3 shows the relationship between step number and correct rate of services' searching

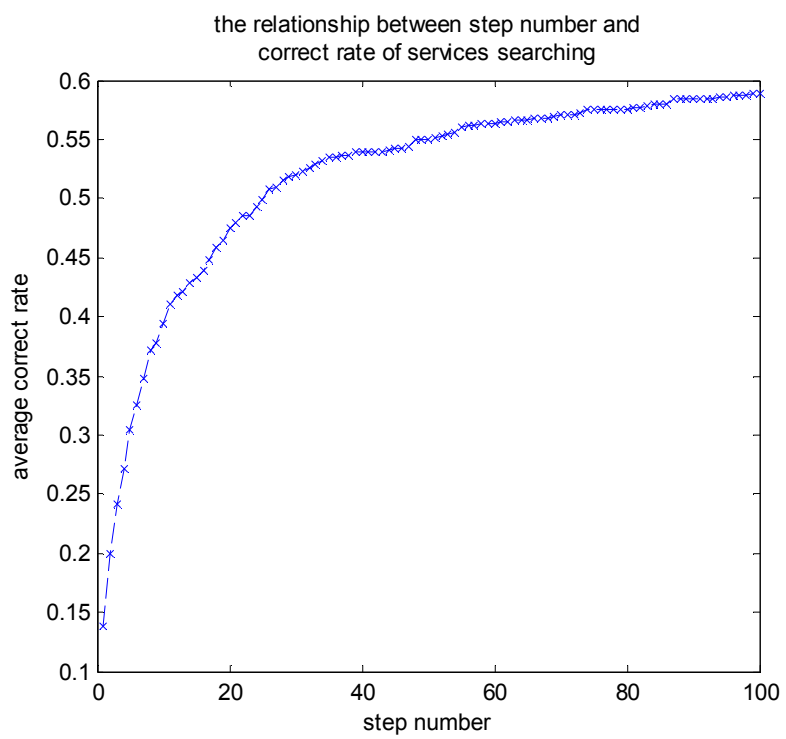

Figure 3 the relationship between step number and correct rate of services' searching

As figure 3 shows, with the increasing of the step number and algorithm executing, the correct rate of service's searching is increasing. It means that with executing of algorithm, the algorithm is searching for optimization services for the users.

V Conclusion

In the paper an improved PSO algorithm is designed to handle the problem of services discover under cloud computing. Though theoretical derivation, the correctness of improved PSO algorithm is proofed. The correctness of the algorithm is also verified by the experiment.

Although the improved the PSO algorithm can handle cloud computing service searching problem, but how to make an algorithm which is more efficient to solve the cloud computing serve searching problem. It remain a question to be discussed.

\section{Reference}

[1] kennedy j, eberhart $\mathrm{r}$ c. Particle swarm optimization [c]// proceedings of ieee international conference on neural networks.piscataway new jersey : institute of electrical and electronics engineers, inc. 1995:1942-1948.

[2]M. Mandal, A. Mukhopadhyay. A multiobjective pso-based approach for identifying non-redundant gene markers from microarray gene expression data. Computing, communication and applications (iccca). pp. 1-6

[3] b. Liu, 1. Wang, and y. H. Jin. An effective pso-based memetic algorithm for flow shop scheduling. Ieee transactions on systems, man, and cybernetics - part b: cybernetics, vol. 37, iss. 1, feb. 2007. pp. 18-27 [4] 1. Messerschmidt, a. P. Engelbrech. Learning to play games using a pso-based competitive learning approach. Evolutionary computation, vol. 8 , iss. 3 , jun. 2004 . pp. $280-288$

[5] K. W. Chau. application of a pso-based neural network in analysis of outcomes of construction claims. Automation in construction. vol. 16, 2007, pp. 642-646

[6] Mohamed Ahmed Mohandes. Modeling global solar radiation using Particle Swarm Optimization (PSO). Solar Energy. vol. 86, iss. 11, nov. 2012 , pp. $3137-3145$

[7] J. Yu, S. Wang, 1. Xi. Evolving artificial neural networks using an improved pso and dpso. Neurocomputing. vol. 71, 2008, pp. 1054 1060

[8]C.-L. Huang, J.-F. Dun. A distributed pso - svm hybrid system with feature selection and parameter optimization. Applied soft computing. vol. 8, iss. 4, september 2008 , pp. $1381-1391$

[9] M. Mandal, A. Mukhopadhyay. A multiobjective PSO-based approach for identifying non-redundant gene markers from microarray gene expression data. Computing. Communication and Applications (ICCCA). pp. 1-6

[10] Archana Sarangia, Rabi Kumar Mahapatrab, Siba Prasada Panigrahi.DEPSO and PSO-QI in digital filter design. Expert Systems with Applications. Vol. 38, Iss. 9, Sep. 2011 .pp. 10966-10973

[11] J. L. Fernandez-Martınez, E. G.-Gonzalo Stochastic Stability Analysis of the Linear Continuous and Discrete PSO Models. Evolutionary Computation. Vol. 15 , Iss. 3,Jun. 2011. pp. 405 - 423

[12] .A. Sarangi a, R. K. Mahapatra b, S. Prasada Panigrahi. DEPSO and PSO-QI in digital filter design. Expert Systems with Applications. Vol. 38, 2011.pp. 10966 - 10973

[13] A. Chandera,A. Chatterjeec, P. Siarrya,. A new social and momentum component adaptive PSO algorithm for image segmentation. Expert Systems with Applications. Expert Systems with Applications Vol. 38, 2011.pp. $4998-5004$

[14] Kennedy J, Eberhart R C. A discrete binary version of the particle swarm algorithm $[\mathrm{C}] / /$ Proceedings of the IEEE International Conference on Systems, Man and Cybernetics. Orlando: IEEE Inc, 1997 pp. 4104 -4108 .

[15] M. Armbrust, A. Fox, R. Griffith. A view of cloud computing. Communications of the acm. Vol. 53, iss. 4, april 2010, pp. 50-58 